\title{
Clinical outcome of robot-assisted residual mass resection in metastatic nonseminomatous germ cell tumor
}

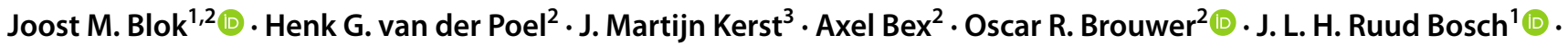 \\ Simon Horenblas ${ }^{2} \cdot$ Richard P. Meijer $^{1}\left[{ }^{10}\right.$
}

Received: 20 July 2020 / Accepted: 31 August 2020 / Published online: 21 September 2020

(c) The Author(s) 2020

\begin{abstract}
Purpose To evaluate the outcome of robot-assisted residual mass resection (RA-RMR) in nonseminomatous germ cell tumor (NSGCT) patients with residual tumor following chemotherapy.

Patients and methods Retrospective medical chart analysis of all patients with NSGCT undergoing RA-RMR at two tertiary referral centers between January 2007 and April 2019. Patients were considered for RA-RMR in case of a residual tumor between 10 and $50 \mathrm{~mm}$ at cross-sectional computed tomography (CT) imaging located ventrally or laterally from the aorta or vena cava, with normalized tumor markers following completion of chemotherapy, and no history of retroperitoneal surgery. Results A total of 45 patients were included in the analysis. The Royal Marsden stage before chemotherapy was IIA in $13(28.9 \%)$, IIB in $16(35.6 \%)$, IIC in $3(6.7 \%)$ and IV in 13 patients (28.9\%). The median residual tumor size was $1.9 \mathrm{~cm}$ (interquartile range [IQR] 1.4-2.8; range 1.0-5.0). Five procedures (11.1\%) were converted to an open procedure due to a vascular injury $(n=2)$, technical difficulty $(n=2)$ or tumor debris leakage $(n=1)$. A postoperative adverse event occurred in two patients (4.4\%). Histopathology showed teratoma, necrosis and viable cancer in 29 (64.4\%), 14 (31.1\%), and two patients (4.4\%), respectively. After a median follow-up of 41 months (IQR 22-70), one patient (2.2\%) relapsed in the retroperitoneum. The one- and 2-year recurrence-free survival rate was $98 \%$.

Conclusion RA-RMR is an appropriate treatment option in selected patients, potentially providing excellent cure rates with minimal morbidity. Long-term outcome data are needed to further support this strategy and determine inclusion and exclusion criteria.
\end{abstract}

Keywords Nonseminomatous germ cell tumor $\cdot$ Retroperitoneal lymph node dissection $\cdot$ Robot-assisted retroperitoneal lymph node dissection $\cdot$ Robotic surgery $\cdot$ Testicular cancer $\cdot$ Testicular germ cell tumor

\begin{tabular}{|c|c|c|}
\hline & Abbreviations & \\
\hline & $\mathrm{CT}$ & Computerized tomography \\
\hline & IGCCCG & $\begin{array}{l}\text { International Germ Cell Cancer Col- } \\
\text { laborative Group }\end{array}$ \\
\hline ctronic supplementary material The online version of this & IQR & Interquartile range \\
\hline $\begin{array}{l}\text { article (https://doi.org/10.1007/s00345-020-03437-z) contains } \\
\text { supplementary material, which is available to authorized users. }\end{array}$ & MSKCC & $\begin{array}{l}\text { Memorial Sloan Kettering Cancer } \\
\text { Center }\end{array}$ \\
\hline Joost M. Blok & NSGCT & Nonseminomatous germ cell tumor \\
\hline j.m.blok-3@umcutrecht.nl & TGCT & Testicular germ cell tumor \\
\hline $\begin{array}{l}\triangle \text { Richard P. Meijer } \\
\text { rmeijer6@umcutrecht.nl }\end{array}$ & PC-RPLND & $\begin{array}{l}\text { Postchemotherapy retroperitoneal } \\
\text { lymph node dissection }\end{array}$ \\
\hline Department of Oncological Urology, University Medical & RA-PC-RPLND & $\begin{array}{l}\text { Robot-assisted postchemotherapy retro- } \\
\text { peritoneal lymph node dissection }\end{array}$ \\
\hline Center Utrecht, Utrecht, The Netherlands & RA-RMR & Robot-assisted residual mass resection \\
\hline
\end{tabular}




\section{Introduction}

Approximately one-third of patients who undergo cisplatin-based combination chemotherapy for disseminated nonseminomatous germ cell tumor (NSGCT) have significant residual retroperitoneal disease [1,2]. Histopathological analysis after postchemotherapy retroperitoneal lymph node dissection (PC-RPLND) shows fibrosis or necrosis in $40-50 \%$, teratoma in $30-40 \%$, and viable cancer in $10-20 \%$ of cases $[3,4]$. Since there are currently no validated methods to reliably predict the histology of a residual mass, PC-RPLND remains important in all patients with significant residual disease in NSGCT [5].

There is a debate concerning the anatomical extent of PC-RPLND. Historically, bilateral template-based retroperitoneal lymph node dissection was the standard approach in all patients undergoing PC-RPLND [5]. Heidenreich et al. showed that a modified template decreases morbidity and does not compromise oncological outcome in selected patients [4]. Although a template-based procedure is the standard approach, several centers consider residual mass resection as oncologically equivalent $[6,7]$.

More recently, the minimally invasive approach is gaining recognition in the post-chemotherapy setting. Two large series have shown excellent oncological outcomes after laparoscopic PC-RPLND [8, 9], but high volume series on robotassisted PC-RPLND (RA-PC-RPLND) are still lacking [10]. In the largest series to date, none of the 30 patients undergoing RA-PC-RPLND had retroperitoneal relapse [11]. These promising initial results and the continuous evolvement of surgical techniques and technology suggest that robotic surgery may replace open PC-RPLND in selected patients. On the condition that oncological safety is warranted, this may provide significant benefit to patients. After all, the morbidity of open PC-RPLND is high, while histopathology of the retroperitoneal specimen shows fibrosis or necrosis in a large proportion of patients $[3,4,12,13]$.

Current reports on minimally invasive PC-RPLND mainly concern template-based surgery. We hypothesized that, in selected patients, oncological control can be achieved by robot-assisted residual mass resection (RARMR). In this study, we retrospectively evaluated the results of this approach in two tertiary referral centers.

\section{Patients and methods}

\section{Study design}

After institutional review board approval, we retrospectively reviewed the medical charts for all NSGCT patients who underwent post-chemotherapy RA-RMR in two tertiary referral centers between January 2007 and April 2019.

Work-up prior to surgery included abdominopelvic computed tomography (CT) scanning and measurement of serum tumor markers ( $\alpha$-fetoprotein, human chorionic gonadotropin and lactate dehydrogenase). All treatment options were discussed by a multidisciplinary panel consisting of a urological oncologist, medical oncologist, radiologist, radiation oncologist and genitourinary pathologist. Patients were considered for RA-RMR in case of one or two residual tumors between 10 and $50 \mathrm{~mm}$ at cross-sectional CT imaging located ventrally or laterally from the aorta or vena cava, with normalized tumor markers following completion of chemotherapy, and no history of retroperitoneal surgery.

\section{Surgical technique}

Patients were positioned in the flank position contralateral of the residual tumor. Exact port placement depended on the location of the residual tumor and the surgeon's preference. In general, a four-port diamond-shaped method was used. The camera port was placed in the paramedian line 3-4 cm cranial to the umbilicus and three additional ports were placed in the upper quadrant, lower quadrant and flank, including an assistant port. In some cases, a fifth port was placed subcostally in the midline.

The surgical resection was not template-based, with the individual extent of the resection adhering to the location of the metastases prior to chemotherapy and the location of the residual tumor (Fig. 1). Any mass in addition to the lesion defined on presurgical CT suspicious for residual tumor that was noticed during surgery was resected as well as lymph nodes in the vicinity and the remnant testicular vessels.

\section{Follow-up}

Follow-up was performed according to current guidelines of the European Society for Medical Oncology. In general, this consisted of monthly clinical examinations and evaluations of serum tumor markers in the first year. After the first year, the frequency of follow-up was gradually reduced every year. Abdominal/thoracic CT scanning was done at least three times (after 6,12 and 24 months).

\section{Results}

Out of a total of 208 RPLNDs, 67 RA-RMRs were performed. Twenty-two patients were excluded from the current analysis, because (a) they were not treated with chemotherapy prior to surgery $(n=15)$, (b) the operative report was missing $(n=2)$, (c) tumor markers were elevated at time of 

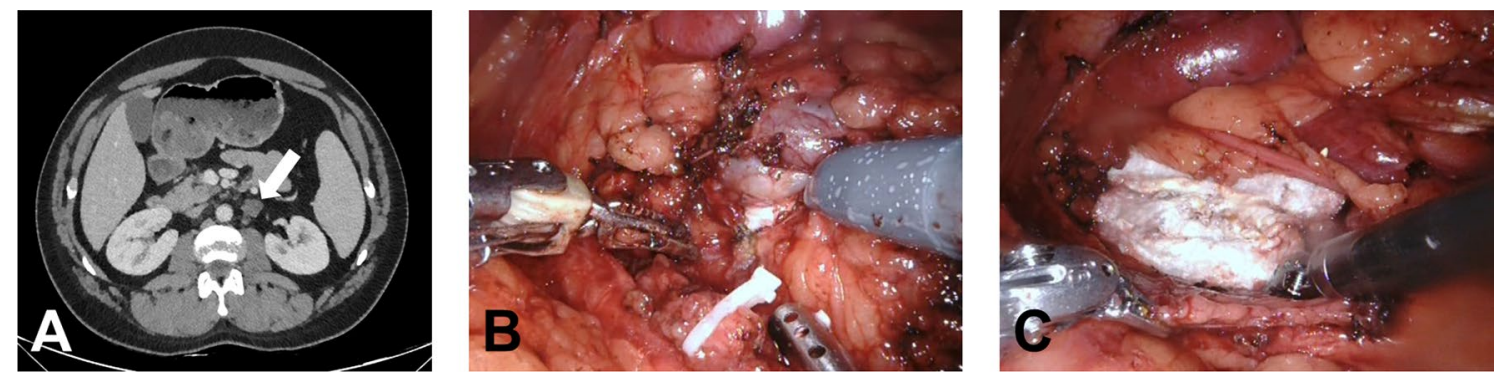

Fig. 1 Computerized tomography scan and intraoperative images of patient undergoing RA-RMR. This patient had a residual tumor (short axis $1.3 \mathrm{~cm}$ ) in the left para-aortal region. Histopathology showed a $3 \mathrm{~cm}$ large teratoma. a Axial abdominal CT scan after chemotherapy with a residual tumor in the left para-aortal region (arrow). b Intra-

surgery $(n=2),(d)$ no NSGCT primary $(n=2)$ or (e) history of prior RPLND $(n=1)$. The remaining 45 patients were included in the analysis (Table 1).

In $71 \%$ of patients, the residual tumor was located in the left para-aortal region. Thirty-eight patients $(84.4 \%)$ had a solitary tumor on preoperative imaging. Five patients (11.1\%) had two nodes and one patient $(2.2 \%)$ had five nodes. The median tumor size was $1.9 \mathrm{~cm}$ (interquartile range [IQR] 1.4-2.8; range 1.0-5.0).

\section{Adverse events}

An intra-operative adverse event was recorded in five patients (11.1\%; Table 1). Two vascular injuries occurred: one renal artery injury and one inferior mesenteric artery injury. Both events required conversion to an open procedure. In two patients, debris leaked from the residual tumor, which required conversion to an open procedure in one case. The fifth patient had a splenic injury, most likely due to excessive traction. No bleeding was observed and the injury was coagulated with a bipolar coagulator.

In addition to the three patients who required a conversion due to an intra-operative adverse event, two patients required conversion to an open procedure due to technical difficulties. One patient had a retro-aortic node adhesive to the surrounding tissue which could not be resected during robotic surgery. The node was successfully resected after conversion. The second patient had two residual tumors: one para-aortic node and one node adjacent to the left common iliac vein. The surgeon was able to resect the para-aortic node during the robot-assisted procedure, but resection of the para-iliacal tumor was unsuccessful. After midline laparotomy, the para-iliacal tumor (sized $4 \times 3 \times 2.5 \mathrm{~cm}$ ) was successfully resected. Palpation of the para-aortal region revealed two additional small nodes which were resected and were confirmed to contain teratoma at histopathology. operative image with the tumor still in situ. c Intra-operative image after the tumor has been resected and a Surgicel has been placed in the retroperitoneum. In images (b) and (c) it is clear that the surrounding nodes and fat are not resected

Two patients (4\%) had a postoperative adverse event Clavien-Dindo grade $\geq 2$. One patient was readmitted 22 days after surgery for a $9 \mathrm{~cm}$ large lymphocele with urinary tract obstruction and secondary pyelonephritis. He was treated with intravenous antibiotics (grade 2 complication). The second patient too was readmitted with a lymphocele six days after surgery (four days after hospital discharge). A drain was placed and a medium-chain triglyceride diet was prescribed (grade 3a).

\section{Histology}

The median number of resected nodes was three (IQR 1-6). The retroperitoneal specimen showed teratoma, necrosis and viable cancer in $29(64 \%), 14(31 \%)$, and 2 patients (4\%), respectively. Since the amount of viable cancer was $<10 \%$ in both patients, they were not treated with additional chemotherapy.

\section{Follow-up}

The median follow-up of the entire cohort was 41 months (IQR 22-70). Follow-up was shorter than 1 year in three patients, who preferred to have their follow-up visits at the referring hospital. Based on only one patient with disease progression, the 1- and 2-year relapse-free survival rates were $98 \%$.

One patient had disease progression with elevated tumor markers. The CT-scan of this patient, prior to RA-RMR, showed a $1.5 \mathrm{~cm}$ large residual tumor cranial to the left renal vessels. The CT-scan 3 months after surgery showed a $2.9 \mathrm{~cm}$ large para-aortic node at the same location, which suggests that the residual tumor was overlooked during surgery and not adequately resected. In addition, a $2.9 \mathrm{~cm}$ large node in the interaortocaval region was found. A CTscan prior to chemotherapy had shown minimal growth of small interaortocaval nodes, but there was no residual 
Table 1 Patient characteristics and outcome

\begin{tabular}{|c|c|}
\hline Number of patients & 45 \\
\hline Median age at surgery, years $(I Q R)$ & $29(23-36)$ \\
\hline \multicolumn{2}{|l|}{ Primary tumor side, $n(\%)$} \\
\hline Left & $32(71.1)$ \\
\hline Right & $13(28.9)$ \\
\hline \multicolumn{2}{|l|}{ Royal Marsden stage prior to chemo, $n(\%)$} \\
\hline IIA & $13(28.9)$ \\
\hline IIB & $16(35.6)$ \\
\hline IIC & $3(6.7)$ \\
\hline IV & $13(28.9)$ \\
\hline \multicolumn{2}{|l|}{ IGCCCG prognosis category } \\
\hline Good & $38(84.4)$ \\
\hline Intermediate & $6(13.3)$ \\
\hline Poor & $1(2.2)$ \\
\hline \multicolumn{2}{|l|}{ Cycles of platinum-based chemotherapy, $n(\%)$} \\
\hline 3 cycles & $24(53.3)$ \\
\hline 4 cycles & $14(31.1)$ \\
\hline$>4$ cycles & $1(2.2)$ \\
\hline Unknown & $6(13.3)$ \\
\hline Median residual tumor size, $\mathrm{cm}(I Q R)$ & $1.9(1.4-2.8)$ \\
\hline \multicolumn{2}{|l|}{ Residual tumor location, $n(\%)$} \\
\hline Para-aortic & $32(71.1)$ \\
\hline Para-caval & $3(6.7)$ \\
\hline Interaortocaval & $10(22.2)$ \\
\hline Median operative time, mins $(I Q R)$ & $134(100-174)$ \\
\hline Median intraoperative blood loss, $m l(I Q R)$ & $50(5-110)$ \\
\hline Intraoperative adverse events, $n(\%)$ & $5(11.1)$ \\
\hline Vascular lesion & $2(4.4)$ \\
\hline Debris leakage & $2(4.4)$ \\
\hline Spleen lesion & $1(2.2)$ \\
\hline Conversions to open surgery, $n(\%)$ & $5(11.1)$ \\
\hline Technical difficulty & $2(4.4)$ \\
\hline Vascular lesion & $2(4.4)$ \\
\hline Debris leakage & $1(2.2)$ \\
\hline Postoperative complication, $n(\%)$ & $2(4.4)$ \\
\hline Clavien-Dindo Grade 2 & $1(2.2)$ \\
\hline Clavien-Dindo Grade 3a & $1(2.2)$ \\
\hline Median length of hospitalization, days (range) & $2(1-3)$ \\
\hline \multicolumn{2}{|l|}{ Retroperitoneal histology, $n(\%)$} \\
\hline Necrosis / fibrosis & $14(31.1)$ \\
\hline Teratoma & $29(64.4)$ \\
\hline Viable cancer & $2(4.4)$ \\
\hline Median length of follow-up, months (IQR) & $41(22-70)$ \\
\hline Relapse, $n(\%)$ & $1(2.2)$ \\
\hline \multicolumn{2}{|l|}{ Survival status, $n(\%)$} \\
\hline No evidence of disease & $43(95.6)$ \\
\hline Died of other causes & $2(4.4)$ \\
\hline
\end{tabular}

IGCCCG International Germ Cell Cancer Collaborative Group, IQR interquartile range tumor visible in the interaortocaval region after completion of chemotherapy. Subsequent treatment with salvage chemotherapy and open RPLND was successful and he had no evidence of disease after 83 months of follow-up.

None of the patients died of disease but two patients died of other causes. One patient died 11 months after surgery due to acute leukemia. Another patient died of renal cell carcinoma, more than 4 years after surgery.

\section{Discussion}

We report the perioperative and oncologic outcomes in a series of 45 selected NSGCT patients undergoing RARMR. Two patients (4.4\%) had a postoperative complication Clavien-Dindo grade $\geq 2$ with short admission time and one patient $(2.2 \%)$ had disease progression in the retroperitoneum. After a median follow-up of more than 3 years, none of the patients had evidence of disease.

Patients with a residual tumor after chemotherapy for disseminated NSGCT form a unique group of cancer patients. They are relatively young and long-term survival is expected in most cases [14]. Although surgical resection of viable cancer is important, histopathological examination of the retroperitoneal specimen shows necrosis in most patients [4, 15]. In addition, the presentation of patients with testicular cancer is changing. The proportion of patients initially presenting with low-stage disease is increasing and systemic chemotherapy is applied more often in patients with lowvolume retroperitoneal metastases [16, 17]. Non-cancer histology is especially common in patients with a small residual lesion [15]. These aspects highlight the increasing importance of the reduction of treatment-associated morbidity and shift the focus of testicular germ cell tumor (TGCT) treatment to a more patient-tailored approach.

Maintaining oncological efficacy is an important prerequisite for the adoption of a minimally invasive approach and several series on minimally invasive PC-RPLND have shown promising results (Supplementary Table 1) [7-9, 11, 18-20]. Steiner et al. reported on 100 patients that were treated with a unilateral $(n=71)$ or bilateral $(n=29)$ laparoscopic template dissection [9]. Patient characteristics were relatively favorable, since the largest tumor diameter was $<1 \mathrm{~cm}$ in $51 / 100$ patients. Only one relapse (outside the surgical field) was observed after a mean follow-up of $>5$ years.

Another key study is a series of 67 patients by Nicolai et al. [8]. Contrary to the series by Steiner et al. only patients with a clinically significant residual tumor $(1-5 \mathrm{~cm})$ were eligible. Although the median follow-up was only 21 months, none of the patients relapsed. These promising findings are supported by a recent systematic review, which found a weighted average retroperitoneal relapse rate of minimally invasive PC-RPLND of only $1.7 \%$ [10]. 
For RA-PC-RPLND specifically, the data on oncological safety are not yet mature enough to draw firm conclusions $[10,21]$. In the largest cohort to date, $\mathrm{Li}$ et al. retrospectively analyzed the outcome of 30 patients undergoing template-based RA-PC-RPLND and compared this with a cohort of patients treated with open resection [11]. None of the patients in the robot-assisted group relapsed in the retroperitoneum.

Several studies have shown that completeness of the residual tumor resection is an important factor in oncological outcome [22, 23]. Fléchon et al. reported the results of 151 patients treated with open PC-RPLND between 1992 and 2002 with the aim to determine whether conformity to the recommendations of the Memorial Sloan Kettering Cancer Center (MSKCC) and completeness of the resection are associated with oncological outcome [22]. Of the 70 patients with a complete resection according to the MSKCC recommendations, only two patients $(2.9 \%)$ had a retroperitoneal relapse. In the group of 58 patients with a complete resection, but not according to the MSKCC recommendations, three patients $(5.2 \%)$ had a retroperitoneal relapse. If patients with an incomplete resection are also considered, thirteen out of 81 patients with a compliant but incomplete resection or with a non-compliant complete or incomplete resection relapsed in the retroperitoneum (16\%). This corresponded to an event-free survival probability at 10 years of $72 \%$, compared to $85 \%$ for patients with compliant and complete resection. It should be noted that the initial tumor was $\geq 5 \mathrm{~cm}$ in fourteen out of fifteen patients with retroperitoneal relapse. In our series, none of the patients had a residual tumor $>5 \mathrm{~cm}$. Nevertheless, this study shows that conformity to the guidelines and completeness of the resection might have an effect on oncological outcome [22].

In another large series of patients undergoing open RMR, seven out of 97 patients with macroscopically complete resection (7\%) suffered from retroperitoneal relapse [6]. As with the study by Fléchon et al., patient characteristics were relatively worse compared to our cohort, since more than half of patients had a residual tumor $>4 \mathrm{~cm}$. Both studies show that RMR may not be an appropriate approach in patients with large residual tumors.

In a randomized comparison of chemotherapeutic regimens, complete resection was mandatory without stating the extent of the template [24]. Four out of 100 patients with normalized tumor markers and nonviable histology of residual tumor (4\%) relapsed. In the group with normalized tumor markers and viable histology of residual tumor, four out of eleven patients (36\%) relapsed. In our series, RA-RMR was only considered in those cases, where complete resection of the residual lesion was considered possible.

The literature on minimally invasive RMR is scarce. Öztürk et al. described the results of laparoscopic RMR in a series of 89 patients treated between 2005 and 2015 [7].
Eight patients (9\%) of the entire cohort relapsed, or four out of 75 procedures that were completed laparoscopically (5\%). This relatively high relapse rate may be explained by the substantial number of patients with vital cancer in the retroperitoneum: $16 \%$ versus $4.4 \%$ in our cohort. In addition, three of the relapsed patients had interaortocaval tumor spread and two had contralateral tumor spread, which would have justified a bilateral dissection according to the Heidenreich criteria [4]. In a series of 12 patients undergoing RA-PC-RPLND by Kamel et al., three patients were treated with RA-RMR [20]. None relapsed after a follow-up of 5, 22 , and 30 months.

In our cohort, one patient had tumor progression. This was partly due to an incomplete resection, but also due to a retroperitoneal relapse in the interaortocaval region outside the surgical field. If this patient would have been treated with a template-based approach, this probably would have been a left-sided modified template, since interaortocaval dissemination is highly unusual in patients with a left-sided primary tumor [25] and the para-aortic residual tumor was only $1.5 \mathrm{~cm}$. This approach would not have prevented the interaortocaval relapse.

An important benefit of minimally invasive surgery is the improved perioperative outcome, compared to open surgery [8, 9, 26-28]. Robot-assisted surgery has additional benefits such as $360^{\circ}$ movement of instruments, ability of three dimensional vision, better surgeon ergonomics, and accuracy and stability in confined spaces [21,29]. The only major complication in our series was a lymphocele requiring drainage. This is in contrast with several population-based studies on open RPLND, which have reported average complication rates of $\sim 25 \%$ [30, 31].

The duration of follow-up in the present study is relatively long, but it is not long enough to safely rule out any future retroperitoneal relapses. Although rare, relapse after complete remission following chemotherapy is possible even beyond 5 years of follow-up [2, 32, 33].

Several studies have shown that patient outcome after complex cancer surgery is correlated with hospital volume $[10,31,34]$. In patients with advanced TGCT, higher hospital volume is associated with improved survival outcomes [35] and high volume hospitals have fewer post-operative complications and more routine home discharges after RPLND [31]. Therefore, patients with advanced TGCT should be managed at high volume expert centers.

Our study is subject to certain limitations. The major limitation is its retrospective design. There were no strict predefined inclusion and exclusion criteria, which may have introduced bias in patient selection. In addition, postoperative antegrade ejaculation was not routinely recorded, which is an important aspect of retroperitoneal surgery.

It is unlikely that all open procedures will be replaced by a minimally invasive approach. In case of a large residual 
tumor, infiltration or encasement of the large vessels, retroaortic or retro-caval tumor location, or if an additional surgical intervention (e.g., nephrectomy) is indicated, open surgery may still be the preferred approach. At the same time, the criteria for a minimally invasive procedure are dynamic instead of fixed. Surgical techniques, surgeon experience and technological innovations keep evolving, which will expand the indication of the minimally invasive approach. For example, the feasibility of a bilateral template dissection without patient repositioning has already been shown [36] and Aufderklamm et al. have reported laparoscopic PC-RPLND with vascular reconstruction in patients with a residual tumor infiltrating the large vessels [37]. Rapidly developing robot-assisted techniques will expand the indication even further.

RMR has been the standard management for post-chemotherapy resection at our institute since 1979 . Not all residual tumor patients are suitable for RMR instead of template dissection. According to the Heidenreich criteria, patients with contralateral tumor spread, residual tumor $>5 \mathrm{~cm}$ or interaortocaval location should undergo a bilateral instead of unilateral template dissection [4]. Thus, they are also not eligible for RMR.

In addition, patients with multiple enlarged nodes postchemotherapy may have an increased risk of microscopic residual teratoma or vital cancer elsewhere in the retroperitoneum and are preferably treated with a template based procedure. It is also conceivable that the extent of the tumor prior to chemotherapy plays an important role. Pre-chemotherapy retroperitoneal nodal size and presence of visceral metastases are associated with relapse after PC-RPLND [3]. Patients with supradiaphragmatic node involvement or multiple tumors prior to chemotherapy may also have an increased risk of residual tumor beyond what is visible on post-chemotherapy CT-scans.

In summary, RA-RMR may be an appropriate treatment option in patients with a single tumor in the primary landing zone which has not extended beyond $5 \mathrm{~cm}$ in diameter since initial diagnosis. However, further studies are necessary to establish the inclusion and exclusion criteria for a more limited dissection.

RA-RMR encompasses two developments: RMR instead of template resection and robot-assisted surgery instead of open surgery. It is important to bear in mind that there are currently no high-volume long-term data on either development. Since RA-RMR is a more limited approach than conventional PC-RPLND, sufficient follow-up is especially important. At the very least, patients should be considered as if they have been treated with a template-based PC-RPLND and thus followed for 5 years. However, it could be the case that patients need to be followed for a longer period of time (e.g., up to 10 years), because they underwent a more limited resection. This is an important topic for further research.

\section{Conclusion}

RA-RMR may be an appropriate treatment option in selected patients, potentially providing excellent cure rates with minimal morbidity at intermediate follow-up. Long-term outcome data are needed to further support this strategy and determine inclusion and exclusion criteria.

Author contributions JM Blok: Project Development, Data Collection, Data Analysis, Manuscript Writing. HG Van der Poel: Data Collection, Manuscript Editing. JM Kerst: Data Collection, Manuscript Editing. A Bex: Data Collection, Manuscript Editing. OR Brouwer: Data Collection, Manuscript Editing. JLHR Bosch: Project Development, Data Collection, Manuscript Editing. S Horenblas: Project Development, Data Collection, Data Analysis, Manuscript Editing. RP Meijer: Project Development, Data Collection, Data Analysis, Manuscript Editing.

Funding Not applicable.

\section{Compliance with ethical standard}

Conflict of interest The authors declare that they have no conflict of interest.

Ethics approval Ethical approval was waived by the local Ethics Committee of both centers in view of the retrospective nature of the study and all the procedures being performed were part of the routine care.

Consent to participate Not applicable.

Consent for publication Not applicable.

Open Access This article is licensed under a Creative Commons Attribution 4.0 International License, which permits use, sharing, adaptation, distribution and reproduction in any medium or format, as long as you give appropriate credit to the original author(s) and the source, provide a link to the Creative Commons licence, and indicate if changes were made. The images or other third party material in this article are included in the article's Creative Commons licence, unless indicated otherwise in a credit line to the material. If material is not included in the article's Creative Commons licence and your intended use is not permitted by statutory regulation or exceeds the permitted use, you will need to obtain permission directly from the copyright holder. To view a copy of this licence, visit http://creativecommons.org/licenses/by/4.0/.

\section{References}

1. Daneshmand S, Albers P, Fosså SD et al (2012) Contemporary management of postchemotherapy testis cancer. Eur Urol 62:867-876. https://doi.org/10.1016/j.eururo.2012.08.014

2. Kollmannsberger C, Daneshmand S, So A et al (2010) Management of disseminated nonseminomatous germ cell tumors With risk-based chemotherapy followed by response-guided postchemotherapy surgery. J Clin Oncol 28:537-542. https:// doi.org/10.1200/JCO.2009.23.0755

3. Carver BS, Serio AM, Bajorin D et al (2007) Improved clinical outcome in recent years for men with metastatic 
nonseminomatous germ cell tumors. J Clin Oncol 25:56035608. https://doi.org/10.1200/JCO.2007.13.6283

4. Heidenreich A, Pfister D, Witthuhn R et al (2009) Postchemotherapy retroperitoneal lymph node dissection in advanced testicular cancer: radical or modified template resection. Eur Urol 55:217-226. https://doi.org/10.1016/j.eururo.2008.09.027

5. Heidenreich A, Paffenholz P, Nestler T, Pfister D (2019) Management of residual masses in testicular germ cell tumors. Expert Rev Anticancer Ther 19:1-10. https://doi. org/10.1080/14737140.2019.1580146

6. Schmidt AH, Høyer M, Jensen BFS, Agerbaek M (2018) Limited post-chemotherapy retroperitoneal resection of residual tumour in non-seminomatous testicular cancer: complications, outcome and quality of life. Acta Oncol (Madr) 57:1084-1093. https://doi.org/10.1080/0284186X.2018.1449249

7. Öztürk Ç, Been LB, van Ginkel RJ et al (2019) Laparoscopic resection of residual retroperitoneal tumor mass in advanced nonseminomatous testicular germ cell tumors; a feasible and safe oncological procedure. Sci Rep 9:15837. https://doi.org/10.1038/ s41598-019-52109-5

8. Nicolai N, Cattaneo F, Biasoni D et al (2016) Laparoscopic postchemotherapy retroperitoneal lymph-node dissection can be a standard option in defined nonseminomatous germ cell tumor patients. J Endourol 30:1112-1119. https://doi.org/10.1089/ end.2016.0458

9. Steiner H, Leonhartsberger N, Stoehr B et al (2013) Postchemotherapy laparoscopic retroperitoneal lymph node dissection for low-volume, stage ii, nonseminomatous germ cell tumor: first 100 patients. Eur Urol 63:1013-1017. https://doi.org/10.1016/j.eurur o.2012.09.036

10. Haarsma R, Blok JM, van Putten K, Meijer RP (2020) Clinical outcome of post-chemotherapy retroperitoneal lymph node dissection in metastatic nonseminomatous germ cell tumour: A systematic review. Eur J Surg Oncol 46:999-1005. https://doi. org/10.1016/j.ejso.2020.02.035

11. Li R, Duplisea JJ, Petros FG et al (2019) Robotic postchemotherapy retroperitoneal lymph node dissection for testicular cancer. Eur Urol Oncol. https://doi.org/10.1016/j.euo.2019.01.014

12. Albers P, Weissbach L, Krege S et al (2004) Prediction of necrosis after chemotherapy of advanced germ cell tumors: results of a prospective multicenter trial of the German Testicular Cancer Study Group. J Urol 171:1835-1838. https://doi.org/10.1097/01. ju.0000119121.36427.09

13. Blok JM, Meijer RP, van der Poel HG et al (2020) Additional surgical procedures and perioperative morbidity in post-chemotherapy retroperitoneal lymph node dissection for metastatic testicular cancer in two intermediate volume hospitals. World J Urol. https ://doi.org/10.1007/s00345-020-03229-5

14. Kvammen O, Myklebust TA, Solberg A et al (2016) Long-term relative survival after diagnosis of testicular germ cell tumor. Cancer Epidemiol Biomarkers Prev 25:773-779. https://doi. org/10.1158/1055-9965.EPI-15-1153

15. Oldenburg J, Alfsen GCC, Lien HHH et al (2003) Postchemotherapy retroperitoneal surgery remains necessary in patients with nonseminomatous testicular cancer and minimal residual tumor masses. J Clin Oncol 21:3310-3317. https://doi.org/10.1200/ JCO.2003.03.184

16. Powles TB, Bhardwa J, Shamash J et al (2005) The changing presentation of germ cell tumours of the testis between 1983 and 2002. BJU Int 95:1197-1200. https://doi.org/10.1111/j.1464410X.2005.05504.x

17. Saera JM, Urtasun JA, Beveridge RD et al (2006) Epidemiological pattern and time trends in testicular germ-cell tumors: a single institution 20-year experience. Clin Transl Oncol 8:588-593. https ://doi.org/10.1007/s12094-006-0064-2
18. Overs C, Beauval JB, Mourey L et al (2018) Robot-assisted postchemotherapy retroperitoneal lymph node dissection in germ cell tumor: is the single-docking with lateral approach relevant? World J Urol 36:655-661. https://doi.org/10.1007/s00345-018-2177-y

19. Singh A, Chatterjee S, Bansal $P$ et al (2017) Robot-assisted retroperitoneal lymph node dissection: Feasibility and outcome in postchemotherapy residual mass in testicular cancer. Indian J Urol 33:304. https://doi.org/10.4103/iju.IJU_8_17

20. Kamel MH, Littlejohn N, Cox M et al (2016) Post-chemotherapy robotic retroperitoneal lymph node dissection: institutional experience. J Endourol 30:510-519. https://doi.org/10.1089/ end.2015.0673

21. Tselos A, Moris D, Tsilimigras DI et al (2018) Robot-assisted retroperitoneal lymphadenectomy in testicular cancer treatment: a systematic review. J Laparoendosc Adv Surg Tech 28:682689. https://doi.org/10.1089/lap.2017.0672

22. Fléchon A, Tavernier E, Boyle $\mathrm{H}$ et al (2010) Long-term oncological outcome after post-chemotherapy retroperitoneal lymph node dissection in men with metastatic nonseminomatous germ cell tumour. BJU Int 106:779-785. https://doi.org/10.1111/ j.1464-410X.2009.09175.x

23. Carver BS, Cronin AM, Eggener S et al (2010) The total number of retroperitoneal lymph nodes resected impacts clinical outcome after chemotherapy for metastatic testicular cancer. Urology 75:1431-1435. https://doi.org/10.1016/j.urolo gy.2009.11.076

24. Culine S, Kerbrat P, Kramar A et al (2007) Refining the optimal chemotherapy regimen for good-risk metastatic nonseminomatous germ-cell tumors: A randomized trial of the Genito-Urinary Group of the French Federation of Cancer Centers (GETUG T93BP). Ann Oncol 18:917-924. https://doi.org/10.1093/annon $\mathrm{c} / \mathrm{mdm} 062$

25. Weissbach L, Boedefeld EA (1987) Localization of solitary and multiple metastases in stage II nonseminomatous testis tumor as basis for a modified staging lymph node dissection in stage I. J Urol 138:77-82. https://doi.org/10.1016/S0022-5347(17)42997-1

26. Poulakis V, Skriapas K, de Vries R et al (2006) Quality of life after laparoscopic and open retroperitoneal lymph node dissection in clinical Stage I nonseminomatous germ cell tumor: A comparison study. Urology 68:154-160. https://doi.org/10.1016/j.urolo gy.2006.01.023

27. Rocco NR, Stroup SP, Abdul-Muhsin HM et al (2019) Primary robotic RLPND for nonseminomatous germ cell testicular cancer: a two-center analysis of intermediate oncologic and safety outcomes. World J Urol 38:859-867. https://doi.org/10.1007/s0034 5-019-02900-w

28. Groeben C, Koch R, Nestler T et al (2019) Centralization tendencies of retroperitoneal lymph node dissection for testicular cancer in Germany? A total population-based analysis from 2006 to 2015. World J Urol 17:e1139. https://doi.org/10.1007/s0034 5-019-02972-8

29. Bagrodia A, Raman JD (2009) Ergonomic considerations of radical prostatectomy: physician perspective of open, laparoscopic, and robot-assisted techniques. J Endourol 23:627-633. https:// doi.org/10.1089/end.2008.0556

30. Gerdtsson A, Håkansson U, Törnblom M et al (2019) Surgical complications in postchemotherapy retroperitoneal lymph node dissection for nonseminoma germ cell tumour: a population-based study from the swedish norwegian testicular cancer group. Eur Urol Oncol. https://doi.org/10.1016/j.euo.2019.08.002

31. Yu H, Hevelone ND, Patel S et al (2012) Hospital surgical volume, utilization, costs and outcomes of retroperitoneal lymph node dissection for testis cancer. Adv Urol 2012:1-8. https://doi. org/10.1155/2012/189823

32. Ehrlich Y, Brames MJ, Beck SDW et al (2010) Long-term follow-up of cisplatin combination chemotherapy in patients with 
disseminated nonseminomatous germ cell tumors: is a postchemotherapy retroperitoneal lymph node dissection needed after complete remission? J Clin Oncol 28:531-536. https://doi. org/10.1200/JCO.2009.23.0714

33. Oldenburg J, Alfsen GC, Wæhre H, Fosså SD (2006) Late recurrences of germ cell malignancies: A population-based experience over three decades. Br J Cancer 94:820-827. https://doi. org/10.1038/sj.bjc.6603014

34. Bilimoria KY, Bentrem DJ, Feinglass JM et al (2008) Directing surgical quality improvement initiatives: comparison of perioperative mortality and long-term survival for cancer surgery. J Clin Oncol 26:4626-4633. https://doi.org/10.1200/JCO.2007.15.6356

35. Woldu SL, Matulay JT, Clinton TN et al (2018) Impact of hospital case volume on testicular cancer outcomes and practice patterns. Urol Oncol Semin Orig Investig 36:14.e7-14.e15. https://doi. org/10.1016/j.urolonc.2017.08.024
36. Stepanian S, Patel M, Porter J (2016) Robot-assisted laparoscopic retroperitoneal lymph node dissection for testicular cancer: evolution of the technique. Eur Urol 70:661-667. https://doi. org/10.1016/j.eururo.2016.03.031

37. Aufderklamm S, Todenhöfer T, Hennenlotter J et al (2014) Postchemotherapy laparoscopic retroperitoneal lymph node dissection for nonseminomatous germ cell tumors infiltrating the great vessels. J Endourol 28:668-674. https://doi.org/10.1089/ end.2013.0755

Publisher's Note Springer Nature remains neutral with regard to jurisdictional claims in published maps and institutional affiliations. 